\title{
¿Tsunamis en Cuba?
}

\author{
M. O. COTILLA RODRÍGUEZ \\ Departamento de Física de la Tierra, Astronomía y Astrofísica 1 \\ Facultad de Ciencias Físicas, Universidad Complutense de Madrid. \\ macot@fis,ucm.es
}

Recibido: $10 / 06 / 2011$

Aceptado: 20/06/2011

\begin{abstract}
Resumen
Cuba como estructura neotectónica del sur de la placa de Norteamérica ha tenido tres tsunamis. Uno de ellos [local] ocurrió en la zona centro-septentrional [1931.10.01, falla Nortecubana], el otro fue un teletsunami [1755.11.01, del suroeste de la Península Ibérica] que afectó a la bahía de Santiago de Cuba, y el tercero aconteció en 1867.11.18 [M=7,5], por la fuente regional de Islas Vírgenes, que produjo oleaje en la región oriental cubana. Este tsunami se originó al nordeste de Puerto Rico que en 1918.10.11 produjo, con otro terremoto de igual magnitud y en unas coordenadas similares, un tsunami que no afectó a Cuba. Las informaciones sobre la influencia del tsunami regional de 1946.08.08 del noreste de la República Dominicana [Matanzas] en Cuba Occidental-Norte [playas Guanabo-Baracoa] son contrarias a lo esperado con la propagación de las ondas. El evento local de 1939.08.15 que se atribuye a Cuba Centro-Septentrional [Cayo Francés con M=8.1] no se corresponde en lo absoluto con la magnitud máxima de los terremotos de esta región y la potencialidad de la falla Nortecubana. Los tsunamis atribuidos a eventos como los de 1766.06 .11 y 1932.02 .03 en la bahía de Santiago de Cuba no están recogidos en los documentos originales de expertos y testigos presenciales. Los tsunamis de Jamaica no han afectado a las costas de Cuba, a pesar de su cercanía. No hay influencia de fuentes tsunamigénicas de las partes meridional y occidental del Caribe, ni del Golfo de México, en Cuba. Se exponen las dudas sobre la no influencia de los tsunamis de Haití y República Dominicana en Guantánamo, bahía que está más cerca y en la misma latitud, e idéntica orientación espacial que la homóloga de Santiago de Cuba, que se dice ha tenido afectaciones. Las cifras de víctimas mortales en el Caribe son diferentes y contradictorias.
\end{abstract}

Palabras clave: Caribe, Cuba, Tsunami.

\section{Tsunamis in Cuba?}

\begin{abstract}
Cuba as neotectonics structure in the southern of the North American plate had three tsunamis. One of them [local] occurred in the Central-Northern region [1931.10.01, Nortecubana fault], the other was a teletsunami [1755.11.01, in the SW of the Iberian Peninsula] that hit the Bay of Santiago de Cuba, and the third took place at 1867.11.18, by the regional source of Virgin Islands, which produced waves in the Eastern Cuban region. This tsunami originated to the NE of Puerto Rico in 1918.10.11, with another earthquake of equal magnitude and at similar coordinates, produced a tsunami that did not affect Cuba. Information on the influence of regional tsunami in 1946.08.08 of the NE of the Dominican Republic [Matanzas] in Northwestern Cuba [beaches Guanabo-Baracoa] is contrary to expectations with the waves' propagation. The local event of 1939.08 .15 attributed to CentralNorthern Cuba [Cayo Francés with $M=8.1$ ] does not correspond at all with the maximum magnitude of earthquakes in this region and the potential of the Nortecubana fault. Tsunamis attributed to events such as 1766.06.11 and 1932.02.03 in the Santiago de Cuba Bay are not reflected in the original documents from experts and eyewitnesses. Tsunamis from Jamaica have not affected the coasts of Cuba, despite its proximity. There is no influence in Cuba of tsunamigenic sources of the southern and western parts of the Caribbean, or the Gulf of Mexico. Set out the doubts as to the influence of tsunamis from Haiti and Dominican Republic at Guantanamo Bay which is closer to and on the same
\end{abstract}


latitude, and spatial orientation than the counterpart of Santiago de Cuba, that had impact. The number of fatalities by authors in the Caribbean is different and contradictory.

Keywords: Caribbean, Cuba, Tsunami.

Referencia normalizada

Cotilla Rodríguez, M. O., (2011). ¿Tsunamis en Cuba? Física de la Tierra Vol. 23 Núm. 1 (2011): 173-197

\section{Introducción}

Cuba desde el punto de vista físico-geográfico está en la región Caribe. Ella es un archipiélago que pertenece al arco de islas de las Antillas Mayores, del que es la mayor. Sin embargo, desde el punto de vista neotectónico Cuba está en la parte meridional de la placa de Norteamérica y colinda al sur con la placa Caribe (Figura 1A). Es decir, ella no pertenece a la placa Caribe como el resto de islas de las Antillas Mayores [Jamaica, La Española, y Puerto Rico]. Así que es un error realizar análisis sobre la sismicidad, la tectónica, los tsunamis, etc. que sustente la idea de que Cuba es parte del Caribe. No obstante, el autor, ha sostenido desde la década de 1980, junto a otros colegas, que la parte oriental de Cuba se diferencia sustancialmente del resto de la isla, y que esa porción sí pertenece al Caribe.

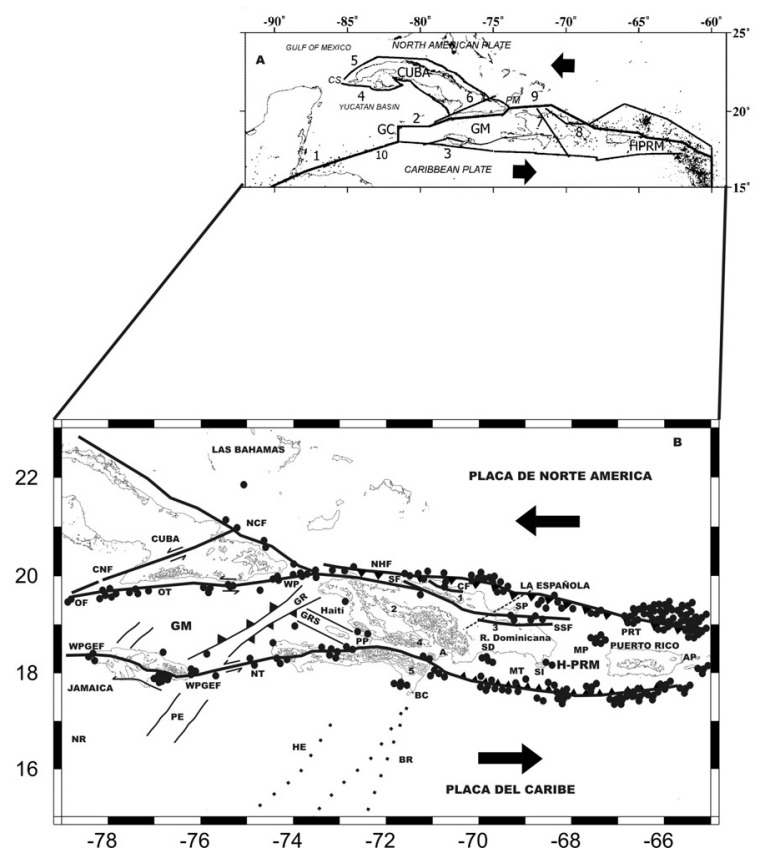

Figura 1.- Esquema tectónico del Caribe.

[A) \{Flechas negras gruesas (sentido del movimiento de las placas); líneas negras= sistemas de fallas principales $(\mathrm{CNF}=$ Cauto-Nipe, $\mathrm{NCF}=$ Nortecubana, $\mathrm{HG}=$ Honduras-Guatemala, $\mathrm{OF}=$ Oriente, $\mathrm{SEF}=$ Septentrional, $\mathrm{SWF}=\mathrm{Swan}, \mathrm{WPGEF}=$ Walton-Platain Garden-Enriquillo); otras estructuras $(\mathrm{CB}=$ Cuenca de Colombia, MP= Pasaje de la Mona, MT: Fosa de los Muertos, NR= Elevado de Nicaragua, 
$\mathrm{OT}=$ Fosa de Oriente, $\mathrm{PBZ}=$ Zona Límite de las Placas, $\mathrm{PRT}=$ Fosa Puerto Rico, $\mathrm{VB}=$ Cuenca de Venezuela, WP $=$ Pasaje de los Vientos); localidades ( $\mathrm{LH}=\mathrm{La}$ Habana, $\mathrm{SC}=$ Santiago de Cuba) $\}$; II= \{Flechas negras gruesas (sentido del movimiento de las placas); círculos negros (epicentros); líneas negras $=$ sistemas de fallas principales $(\mathrm{CF}=\mathrm{Camú}, \mathrm{CNF}=$ Cauto-Nipe, $\mathrm{HG}=$ Honduras-Guatemala, $\mathrm{NCF}=$ Nortecubana, $\mathrm{NHF}=$ Norte de la Española, $\mathrm{OF}=$ Oriente, $\mathrm{SF}=$ Samaná; $\mathrm{SEF}=$ Septentrional, $\mathrm{SWF}=$ Swan, WPGEF $=$ Walton-Platain Garden-Enriquillo); las series de puntos negros indican la localización de las estructuras: $\mathrm{BR}=$ Elevado de Beata, $\mathrm{HE}=$ Escarpe de Hess; Pasajes $(\mathrm{MP}=\mathrm{Mona}$, $\mathrm{WP}=$ de los Vientos); Islas (Cuba, La Española, Jamaica, Puerto Rico); Microplacas (GM= Gonave, HPRM= La Española-Puerto Rico); Fosas $(\mathrm{MT}=$ Muertos, $\mathrm{NT}=$ Navassa, OT= Oriente, $\mathrm{PRT}=$ Puerto Rico); Otras estructuras ( $\mathrm{CB}=$ Cuenca de Colombia, $\mathrm{GR}=$ Elevado de Gonave, GRS= Gonave).]

La región Caribe es conocida por su actividad sísmica y tsunamigénica. Ella fue documentada desde la llegada de los primeros europeos. Esta sismicidad es muy importante en los bordes, que son los límites o contactos entre las placas. Asociados a esos terremotos están algunos eventos de tsunamis. Estos son relativamente frecuentes en las zonas de subducción del oeste y del este, Centroamérica y Antillas Menores, respectivamente.

En el trabajo se presentan y discuten los datos aportados por varios especialistas sobre la ocurrencia de tsunamis en el Caribe (Figura 2B), y en particular en su parte septentrional. Además, se expondrá la posición del autor con relación a los tsunamis que han sido asociados a Cuba.

\section{Notas sobre tsunamis}

El autor considera que es posible obtener una visión general sobre los tsunamis con los siguientes trabajos: Bryant, 2001; Gutenberg, 1939; ITIC, 2004; Kuroiwa, 1985; NOAA, 2004; Satake, 2005; y Sigurdsson, 1996. Ellos han sido empleados aquí.

Los tsunamis son fenómenos naturales derivados, principalmente, de una fuerte actividad sísmica. Ellos se vinculan con las fallas activas de tipo normal, inversa, vertical, y de subducción que provocan la deformación y el levantamiento diferencial de grandes bloques de la corteza. También las erupciones de volcanes submarinos, los deslizamientos de grandes bloques, y los impactos de meteoritos pueden generarles, pero en mucha menor cantidad que las primeramente mencionadas.

Un tsunami es un tren de olas que se desplaza a grandes velocidades, aunque mucho menos que las ondas sísmicas, en las profundidades oceánicas [500-700 $\mathrm{km} / \mathrm{h}$ ]. En estas áreas las amplitudes de las olas son muy bajas y la mayor parte son imperceptibles. Sin embargo, al llegar a las costas o los litorales poco profundos pueden ganar en amplitud [hasta más de $30 \mathrm{~m}$ ], reduciendo la longitud de ondas, y entonces su velocidad de desplazamiento disminuye considerablemente. Esa masa de agua, acorde con el nivel energético y el tipo de fondo y la forma del relieve costero, puede penetrar algunos kilómetros en el interior de un territorio. Evidentemente, son eventos muy peligrosos y hay que estudiarles tanto con datos históricos como instrumentales. 

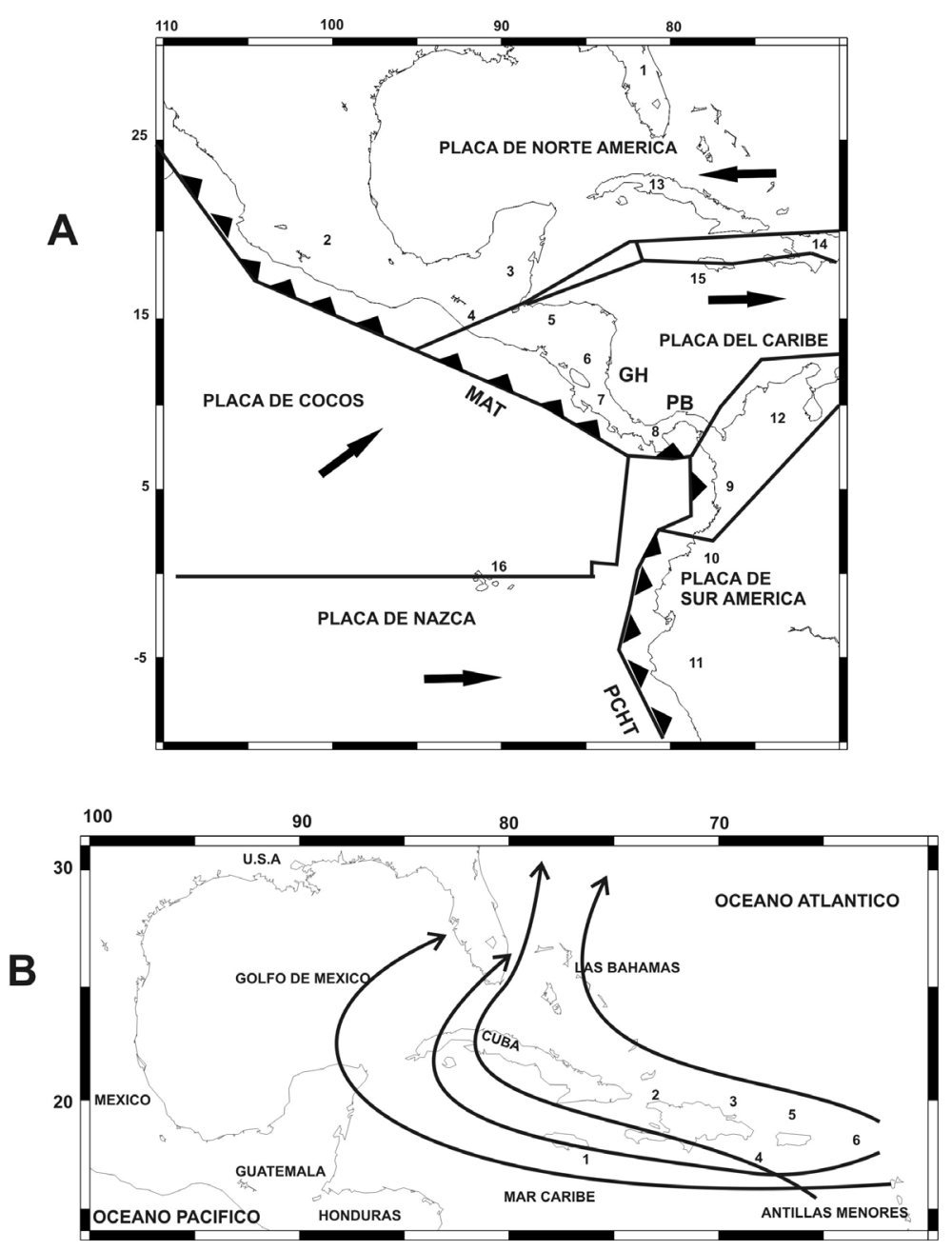

Figura 2A.- Sectores del océano Pacífico, de América del Norte, de América del Sur, de América Central y del Caribe.

Aparecen las placas principales, las zonas de subducción y las principales fallas regionales. Se indican con flechas negras gruesas los sentidos de movimientos predominantes de las placas. El significado de las siglas: I) alfabéticas es: $\mathrm{GH}=$ Golfo de Honduras; MAT= Trinchera Mesoamericana; $\mathrm{PB}=\mathrm{Bloque}$ de Panamá; PCHT= Trinchera de Chile-Perú; II) numéricas son localidades: $1=$ Estados Unidos de Norteamérica; 2= México; 3= Guatemala; 4=El Salvador; 5= Honduras; 6= Nicaragua; 7= Costa Rica; $8=$ Panamá; $9=$ Colombia; $10=$ Ecuador; $11=$ Perú; $12=$ Venezuela; $13=$ Cuba; $14=$ Jamaica; $15=$; $16=$ Islas Galápagos.

Figura 2B.- El Caribe y su entorno.

Aparecen: I) con siglas numéricas: 1= Jamaica; 2= República de Haití; 3= República Dominicana; 4= Fosa de Muertos; 5= Fosa de Puerto Rico; 6= Islas Vírgenes; II) las trayectorias de algunos huracanes y ciclones. 
Los tsunamis se clasifican de forma similar que los terremotos, ya por intensidad o por magnitud. La región con mayor cantidad de tsunamis es la cuenca del Pacífico. En ella se han producido 17 de los 18 tsunamis más fuertes del mundo (Tabla 1). El Caribe es un mar interior del Océano Atlántico, sin embargo éste es mucho menos activo que el Pacífico.

Tabla 1.- Tsunamis más fuertes del mundo.

\begin{tabular}{|c|c|c|c|c|c|}
\hline $\mathbf{N}^{\mathbf{0}}$ & Lugar & Fecha & $\mathbf{N}^{\mathbf{0}}$ & Lugar & Fecha \\
\hline 1 & Chile (Valdivia) & 1837.11 .07 & 10 & Islas Kuriles & 1918.09 .08 \\
\hline 2 & Chile (Arica) & 1868.08 .13 & 11 & Japón & 684.11 .29 \\
\hline 3 & Chile (Iquique) & 1877.05 .10 & 12 & Japón (Sanriku) & 1896.06 .15 \\
\hline 4 & Chile (Atkama) & 1922.11 .10 & 13 & Japón (Peninsula Tsugaru) & 1983.05 .26 \\
\hline 5 & Chile-Perú & 1960.05 .22 & 14 & Japón (Sendai) & 2011.03 .11 \\
\hline 6 & $\begin{array}{c}\text { Ecuador- } \\
\text { Colombia }\end{array}$ & 1906.01 .31 & 15 & Océano Indíco & 2004.12 .26 \\
\hline 7 & Islas Aleutinas & 1946.04 .01 & 16 & Rusia (Kamchatka) & 1841.05 .17 \\
\hline 8 & Islas Aleutinas & 1957.03 .09 & 17 & Rusia (Kamchatka) & 1952.11 .04 \\
\hline 9 & Islas Aleutinas & 1965.02 .04 & 18 & U.S.A. (Alaska) & 1964.03 .28 \\
\hline
\end{tabular}

Otra forma de clasificar a los tsunamis es atendiendo a la distancia de la fuente. En este sentido hay tres tipos: 1) locales $[<100 \mathrm{~km}]$; 2) regionales [100-750 km]; 3) lejanos o teletsunamis $[>750 \mathrm{~km}]$. Todos ellos están en el Caribe.

Las investigaciones de tsunamis son similares a las de terremotos. Esto significa que es posible: 1) realizar búsquedas en hemerotecas y archivos en general con el propósito de configurar un catálogo; 2) acometer trabajos de campo para reconocer sobre el terreno las penetraciones y las afectaciones producidas por los tsunamis; 3) trabajar con perforaciones, trincheras y calas el subsuelo para determinar alteraciones en el perfil de los sedimentos costeros; 4) modelizar matemáticamente las fuentes tsunamigénicas para determinar el alcance de las olas y su peligrosidad. Para este último punto hay muchos resultados desde la década de 1990, pero aquí indicaremos los siguientes: Mercado y McCann (1998), Nagano et al. (1991), Ng et al. (1990), Nuñez-Cornú et al. (2008), Shuto (1991), UNESCO-IOC (1997), y Weissert (1990). El Caribe no ha quedado ajeno a tal tarea (Mercado y McCann, 1998; Weissert, 1990).

\section{Principales características tectónicas de la región Caribe}

En los siguientes trabajos pueden localizar toda la información sobre la tectónica de la región Caribe: Brink et al., 2004; Cotilla, 1998; Cotilla y Córdoba, 2011, 2007; Cotilla y Udías, 1999; y Cotilla et al., 2007, 1997, 1991; Johnston and Thorkelson, 1997; Kellog and Bonini, 1982; Protti et al., 1994; Rubio et al., 1994; y Schwab et al., 1991. No obstante, con el propósito de situar al lector se presenta un resumen con los aspectos más significativos, desde el punto de vista del autor.

El área del Mar Caribe y América Central forma una pequeña placa litosférica acoplada entre las placas de Norte y Suramérica, Cocos y Nazca (Figura 2A). El 
límite norte está constituido por un sistema de fallas activas de desplazamiento lateral a la izquierda (aproximadamente de 2,000 km de extensión) que enlaza en sus extremos a dos zonas de subducción. El contacto oriental se localiza aproximadamente en los $-71^{\circ} \mathrm{O}$. Sin embargo, en el margen septentrional destacan segmentos con convergencia de pequeña magnitud en dirección aproximada N-S. La zona

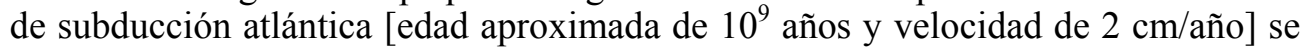
extiende hasta las inmediaciones de Venezuela. La parte oeste se distingue por la subducción de la placa Cocos bajo la placa Caribe y la modificación del perfil y las características de este borde activo. El borde sur de la placa Caribe se extiende por el denominado Cinturón Deformado de Panamá, las fallas El Pilar, Boconó, y otras.

La estructura dominante de la parte norte de la placa Caribe es la fosa Bartlett Caimán. Ésta es una estructura submarina de tipo pull-apart de $\sim 1,000 \mathrm{~km}$ de longitud a partir de un pequeño centro de generación de corteza oceánica de $110 \mathrm{~km}$ de ancho. Ese centro se asume está activo desde el Eoceno Medio [velocidad aproximada de expansión $1.5 \mathrm{~cm} / \mathrm{año}]$.

\subsection{Datos tectónicos de Cuba}

La información empleada proviene de Cotilla et al. (1991). Así, Cuba constituye un megabloque [o microplaca] emergido y en ascenso diferencial entre sus partes que está incorporado tectónicamente al borde meridional de la placa Norteamérica (Figura 1A). Sus particularidades estructurales, complejas y heterogéneas, fueron adquiridas en las dos principales etapas de su desarrollo geológico: orogenia cubana [Eoceno Medio] y neotectónica [Post Eoceno Superior], caracterizadas respectivamente por movimientos compresivos, y movimientos verticales oscilatorios; responsables estos últimos de la diferenciación, la división en bloques del archipiélago y el desarrollo de una cobertura sedimentaria poco deformada que cubre en parte a las estructuras pre neotectónicas, y que se reflejan en el relieve del megabloque. La división en bloques se produjo a partir de los límites tectónicos longitudinales y transversales de la etapa pre Eoceno Superior, como zonas de debilidad aprovechadas por las rupturas nuevas, aunque con otro estilo y tendencia, fundamentalmente vertical. La complejidad de la estructura geológica de Cuba se evidencia en cortes geológicos y geofísicos en que aparecen apiladas, mezcladas y dislocadas diferentemente secuencias rocosas de distintas zonas estructuro-formacionales.

Los datos de sismicidad en Cuba muestran una significativa y regular disminución, desde la zona de contacto de las placas Caribe-Norteamérica en la parte suroriental hacia el interior del territorio [parte occidental] (Figura 3). Esto permite sostener la existencia de dos tipos de sismicidad: entreplacas e interior de placas. Esto se corresponde con la situación neotectónica y permite sostener la existencia de dos unidades independientes: Oriental y Occidental (Figura 3). Estas Unidades mantienen entre ellas una apreciable diferenciación en cuanto a la relación actividad / extensión territorial, destacándose que el valor, de la relación anterior, para la Oriental es muy superior al de la Unidad Neotectónica Occidental; lo cual puede justificarse por su inmediata cercanía a la estructura disyuntiva activa de BartlettCaimán [segmento Swan-Oriente]. 


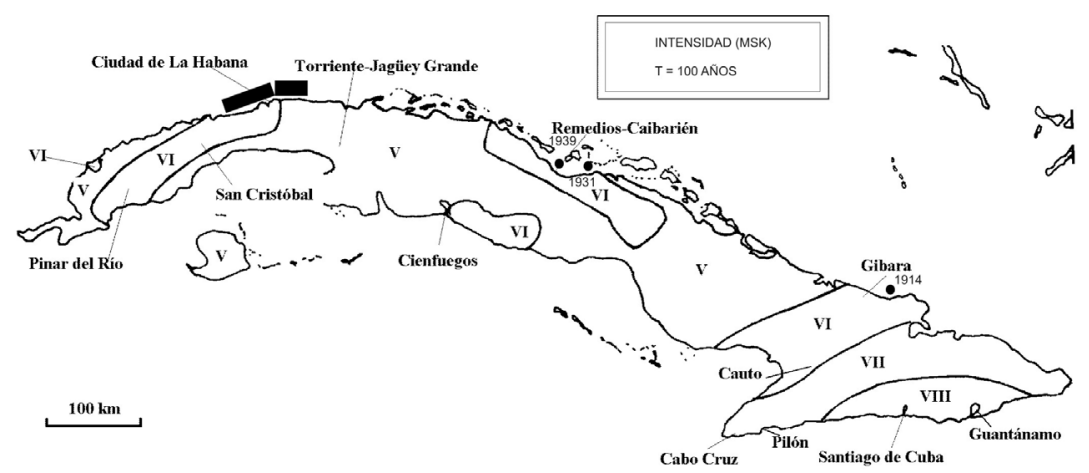

Figura 3.- Mapa de Intensidades Sísmicas de Cuba [T=100 años].

Aparecen: 1) las zonas de intensidades para valores de la escala MSK; 2) las áreas de playas de Guanabo a Baracoa se indican con rectángulos negros; 3) dos epicentros de terremotos para los tsunamis de 1931 y 1939, y el epicentro de 1914 [M=6.2] en Gibara.

\section{Bosquejo sobre los terremotos y tsunamis en el Caribe}

La sismicidad del Caribe (Figuras 1B y 4) ha sido reflejada en no pocos trabajos y entre ellos mencionamos: Álvarez et al., 1999; Ambraseys y Adams, 1996; BRGM, 1990; Cotilla, 2007; Cotilla et al., 1997. Mientras que los datos sobre tsunamis están entre otros en: Affleck, 1809; Arce et al., 1998; BRGM, 1990; Caicedo et al., 1996; Cruz y Wyss, 1983; Fernández et al., 2000, 1999; Grindlay et al., 2005; Heilpin, 1903; Hillebrandt-Andrande, 2005; Hillebrandt-Andrade et al., 2004; HTDB/PAC, 2001; Lander y Whitside, 1997; Lander et al., 2002; Lynch y Bodle, 1948; McNamara et al., 2005, 2005A; Mercado y MCann, 1998; Molina, 1997; Montelieu, 1933; Montero, 1990; Nealon y Dillon, 2001; O'Linghlin y Lander, 2003; Poey, 1855; Reid y Taber, 1919; Robson, 1964; Romero, 1998; Rubio, 1982; Sainte-Claire Deville, 1867; Shubert, 1994; Weissert, 1990; Zahibo y Pelinovsky, 2001.

Los terremotos de las partes occidental y oriental del Caribe están asociados con zonas de subducción. Ellas se diferencian entre sí en cuanto al perfil transversal, la profundidad, la frecuencia, y la magnitud de los terremotos. En este sentido, la parte occidental o del Pacífico, que se corresponde con Centroamérica, es la más activa y tiene volcanes y una importante actividad tsunamigénica (Tabla 2). Pero la parte oriental o del Atlántico, en las Antillas Menores, tiene también potencialidad tsunamigénica (Tablas 3A y 3B). Esas dos partes demuestran tener diferente actividad sísmica con perfiles norte - sur y son fuentes capaces de generar tsunamis que afecten al interior caribeño. 
Tabla 2.- Tsunamis en América Central-Pacífico.

\begin{tabular}{|c|c|c|c|c|c|}
\hline $\mathbf{N}^{\mathbf{0}}$ & Fecha & H (km) & Ms & Lugar & Runup (m) \\
\hline 1 & 1854.08 .05 & 33 & 7.3 & Costa Rica (Golfo Dulce) & \\
\hline 2 & 1884.11 .05 & 100 & 7.5 & Colombia (Acando) & \\
\hline 3 & 1941.12 .05 & & 7.6 & Costa Rica (Dominsal) & 0.2 \\
\hline 4 & 1950.10 .05 & 60 & 7.7 & Costa Rica-Nicaragua- El Salvador & \\
\hline 5 & 1906.01 .31 & & 8.1 & Ecuador-Panama-Costa Rica & 2.5 \\
\hline 6 & 1915.09 .07 & 60 & 7.7 & El Salvador (costa sur) & \\
\hline 7 & 1916.05 .25 & & 7.5 & El Salvador & $>2$ \\
\hline 8 & 1957.03 .10 & & 8.1 & El Salvador (Acajutla) & \\
\hline 9 & 1902.04 .19 & 60 & 7.5 & Guatemala (Ocos) & \\
\hline 10 & 1950.10 .23 & & 7.3 & Guatemala-El Salvador (costa) & \\
\hline 11 & 1960.05 .22 & 32 & 8.5 & Guatemala-El Salvador & \\
\hline 12 & 1844.05 & 30 & 7.4 & Lago Nicaragua & \\
\hline 13 & 1956.10 .24 & & 7.2 & Nicaragua (San Juan Sur) & \\
\hline 14 & 1934.07 .18 & & 7.5 & Panama (Golfo de Chiqui) & 0.6 \\
\hline
\end{tabular}

Tabla 3A.- Tsunamis en Las Antillas Menores-Atlántico (Zahibo y Pelinovsky, 2001).

\begin{tabular}{|c|c|l|c|}
\hline $\mathbf{N}^{\mathbf{0}}$ & Fecha & \multicolumn{1}{|c|}{ Lugar } & Nota \\
\hline 1 & 1751.11 .20 & Antigua & No Lander \\
\hline 2 & 1755.11 .01 & Barbados, Martinica, Dominica, Antigua, Saba, y St.Martin & \\
\hline 3 & 1761.03 .31 & Barbados & \\
\hline 4 & 1767.04 .24 & Barbados y Martinica & No Lander \\
\hline 5 & 1802.03 .19 & Antigua and St. Christopher & \\
\hline 6 & 1823.11 .30 & Saint Pierre Harbour, Martinica & No Lander \\
\hline 7 & $1824.09 .9-13$ & Base Terre, Guadalupe y Plymouth, Montserrat & No Lander \\
\hline 8 & 1831.12 .03 & Trinidad, Antigua y St. Christopher & No Lander \\
\hline 9 & 1837.07 .26 & Martinica & No Lander \\
\hline 10 & 1837.08 .02 & Martinica & No Lander \\
\hline 11 & 1842.02 .17 & Antigua, volcán & \\
\hline 12 & 1843.02 .08 & Guadalupe, Montserrat, y Martinica & No Lander \\
\hline 13 & 1867.11 .18 & Islas Vírgenes & \\
\hline 14 & 1874.03 .11 & Islas Vírgenes & \\
\hline 15 & 1902.05 .05 & Martinica [Mont. Pelée, volcán] & \\
\hline 16 & 1902.05 .07 & Martinica [Mont. Pelée, volcán] & \\
\hline 17 & 1911.03 .03 & Trinidad y Tobago, volcán & \\
\hline 18 & 1969.12 .25 & Barbados & \\
\hline 19 & 1985.03 .16 & Guadalupe & \\
\hline 20 & 1991.04 .22 & Martinica & \\
\hline 21 & 1997.07 .09 & Tobago & \\
\hline 22 & 1997.12 .26 & Montserrat, volcán & \\
\hline
\end{tabular}

Nota: Lander = Lander et al., 2002. 
Tabla 3B.- Tsunamis en Las Antillas Menores-Atlántico (Lander et al., 2002).

\begin{tabular}{|c|c|l|c|}
\hline $\mathbf{N}^{\mathbf{0}}$ & \multicolumn{1}{|c|}{ Fecha } & \multicolumn{1}{|c|}{ Lugar } & Runup (m) \\
\hline 1 & 1690.04 .16 & Islas Vírgenes [Isla Leeward] & $7.0-1.5$ \\
\hline 2 & 1755.11 .01 & $\begin{array}{l}\text { Lisboa: Antillas Holandesas [Saba y St, Martin]; } \\
\text { Antigua, Dominica, Barbados, Martinica; Cuba } \\
\text { [Santiago de Cuba] }\end{array}$ & \\
\hline 3 & 1755.11 .18 & St. Martin & 1.2 \\
\hline 4 & 1761.03 .31 & Barbados & $19.8-0.9$ \\
\hline 5 & 1767.04 .24 & Barbados y Martinica & \\
\hline 6 & 1823.11 .30 & Saint Pierre Harbour, Martinica & \\
\hline 7 & 1842.05 .07 & Islas Vírgenes [St. John] & \\
\hline 8 & 1843.02 .08 & Isla Guadalupe, Antigua & \\
\hline 9 & 1867.11 .18 & $\begin{array}{l}\text { Islas Vírgenes [St. Croix y St. Thomas], Guada- } \\
\text { lupe }\end{array}$ & \\
\hline 10 & 1883.08 .27 & $\begin{array}{l}\text { Indonesia [Krakatoa, volcán]: Islas Vírgenes [St. } \\
\text { Thomas] }\end{array}$ & $0.46-0.12$ \\
\hline 11 & 1902.08 .30 & Martinica [Fort-de-France] & \\
\hline 12 & 1911.03 .03 & Trinidad & \\
\hline 13 & 1918.10 .11 & Puerto Rico: Islas Vírgenes [Tortola] & \\
\hline 14 & 1969.12 .25 & Barbados, Antigua y Dominica & \\
\hline 15 & 1985.03 .16 & Guadalupe [Basse-Terre] & Costa Rica: Islas Vírgenes [St. Croix y Limetree] \\
\hline 16 & 1991.04 .22 & Venezuela: Tobago & \\
\hline 17 & 1997.07 .09 & Ventserrat & \\
\hline 18 & 1997.12 .26 & Mont & \\
\hline
\end{tabular}

Específicamente, el área oriental de Centroamérica, ya en el Mar Caribe, ha tenido algunos eventos tsunamigénicos significativos (Tablas 4A y 4B). También la parte septentrional del Caribe ha sido afectada por algunos terremotos fuertes y tsunamis (Tablas 5A y 5B). Sin embargo, ninguno de ellos fue producido por volcán.

Tabla 4A.- Tsunamis en América Central-Mar Caribe (Lander et al., 2002).

\begin{tabular}{|c|c|l|c|c|}
\hline $\mathbf{N}^{\mathbf{0}}$ & Fecha / Tiempo & \multicolumn{1}{|c|}{ Lugar } & Ms & Runup (m) \\
\hline 1 & 1856.08 .09 & Honduras [Río Patuca, Omoa] & 7.5 & 5.0 \\
\hline 2 & $1916.04 .24 / 08: 02 \mathrm{UT}$ & $\begin{array}{l}\text { Panamá [Almirante, Bocas de } \\
\text { Toro] }\end{array}$ & 7.6 & \\
\hline 3 & $1991.04 .22 / 21: 56 \mathrm{UT}$ & Panamá [Bocas de Toro] & 7.4 & 0.6 \\
\hline
\end{tabular}

Al norte del Caribe hay una importante masa de agua, el Golfo de México. En ella los terremotos han sido muy pocos y siempre de baja energía. Así que su potencialidad sísmica es despreciable. De otra parte, la capacidad tsunamigénica es muy baja, por no decir que nula. 
Tabla 4B.- Tsunamis en América Central-Mar Caribe (Fernández et al., 2000).

\begin{tabular}{|c|c|c|c|c|c|}
\hline $\mathbf{N}^{\mathbf{0}}$ & Fecha & Lugar & Ms & H (km) & Nota \\
\hline 1 & 1798.02 .22 & Costa Rica [Matina] & & & No Lander \\
\hline 2 & 1822.05 .07 & Costa Rica [Matina] & 7.6 & & No Lander \\
\hline 3 & 1539.11 .24 & Golfo de Honduras & & & No Lander \\
\hline 4 & 1825.02. & Golfo de Honduras & 5.5 & & No Lander \\
\hline 5 & 1855.09 .25 & Golfo de Honduras & 6.5 & & No Lander \\
\hline 6 & 1856.08 .04 & Golfo de Honduras & 8.0 & & No Lander \\
\hline 7 & 1976.02 .04 & Golfo de Honduras & 7.5 & 5 & No Lander \\
\hline 8 & 1873.10 .14 & Panamá [Bahía Colón] & & & No Lander \\
\hline 9 & 1882.09 .07 & Panamá [San Blas] & 7.9 & & No Lander \\
\hline 10 & 1904.12 .20 & Panamá [Bocas Toro] & 7.3 & 25 & No Lander \\
\hline 11 & 1916.04 .26 & Panamá [Bocas Toro] & 6.9 & & No Lander \\
\hline 12 & 1991.04 .22 & Panamá [Bocas Toro] & 7.6 & 20 & \\
\hline
\end{tabular}

Nota: Lander = Lander et al., 2002.

En la tabla 6 aparecen los tsunamis de fuentes lejanas que afectaron al Caribe. Mientras que las tablas 7A y 7B contienen los datos de tsunamis que han sido determinados en Venezuela. Y la cantidad de fallecidos, por distintas fuentes, en el Caribe como consecuencia de los tsunamis está en la tabla 8. En este sentido se aprecian no pocas diferencias, para un mismo evento, por lo que tales cifras deben ser estudiadas en detalle a partir de fuentes contemporáneas con los eventos a los que se asocian las pérdidas. Pero, esto escapa a nuestro objetivo.

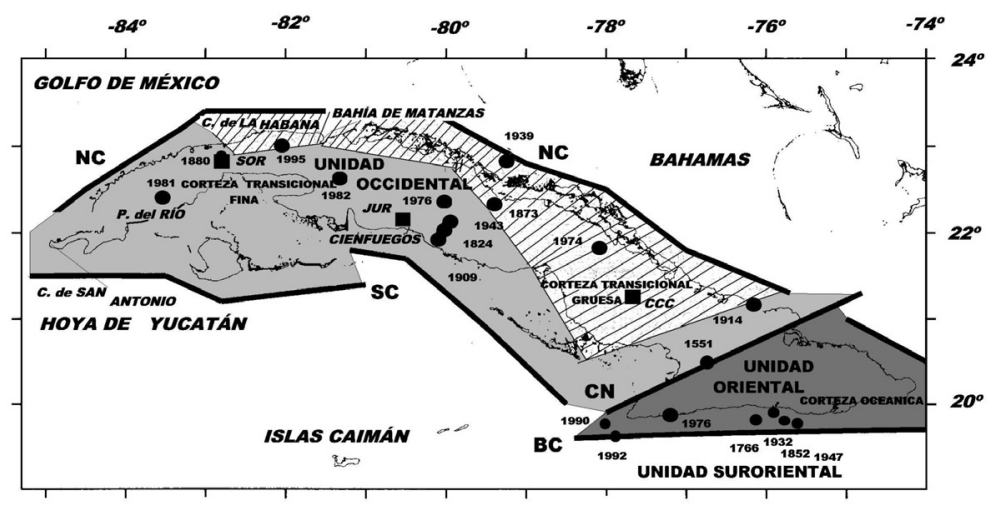

Figura 4.- Cuba.

Aparecen: 1) las tres Grandes Unidades Sismotectónicas [Occidental, Oriental, y Suroriental]; 2) las principales zonas de fallas [Oriente, Nortecubana, Surcubana, y Cauto-Nipe]; 3) los tres tipos de corteza [transicional gruesa, transicional fina, y oceánica]; 4) una selección de epicentros de terremotos [círculos negros, y el año de ocurrencia]; 5) algunas localidades. 
Tabla 5A.- Tsunamis en la parte septentrional del Caribe (Rubio, 1982).

\begin{tabular}{|c|c|c|c|}
\hline $\mathbf{N}^{\mathbf{0}}$ & Fecha / Tiempo & Lugar & Comentario \\
\hline 1 & 1692.06 .07 & Jamaica [Port Royal] & No afectó Cuba \\
\hline 2 & 1751.10.18/15:00 & República Dominicana [Azua] & No afectó Cuba \\
\hline 3 & $1755.11 .01 /-$ & Santiago de Cuba & \\
\hline 4 & 1770.06.03 / 19:15 & Haití [Port-au-Prince] & No afectó Cuba \\
\hline 5 & $1755 /$ & Haití & No afectó Cuba \\
\hline 6 & $1775.03 /$ & República Dominicana & No afectó Cuba \\
\hline 7 & $1780.10 .03 / 22: 00$ & Jamaica [Savanna-la-Mar] & No afectó Cuba \\
\hline 8 & $1787.10 .27 / 14: 20$ & Jamaica [Montego Bay] & No afectó Cuba \\
\hline 9 & 1842.05 .07 & Haití [Port de Paix] & No afectó Cuba \\
\hline 10 & 1852.07 .17 & Santiago de Cuba & \\
\hline 11 & 1867.11 .18 & Islas Vírgenes [St. Thomas] & Afectó Cuba Oriental \\
\hline 12 & 1907.01.14 & Jamaica [Kingston] & No afectó Cuba \\
\hline 13 & $1918.10 .11 / 10: 14$ & Puerto Rico & No afectó Cuba \\
\hline 14 & 1931.10 .01 & $\begin{array}{c}\text { Cuba [Playa Panchita, Rancho } \\
\text { Veloz, Las Villas (Cuba centro } \\
\text { norte)] }\end{array}$ & \\
\hline 15 & 1932.02 .03 & Santiago de Cuba & \\
\hline 16 & 1939.08 .15 & $\begin{array}{l}\text { Cuba [Cayo Francés (Cuba } \\
\text { centro norte)] }\end{array}$ & \\
\hline 17 & 1946.08 .08 & $\begin{array}{l}\text { República Dominicana [Matan- } \\
\text { zas] }\end{array}$ & $\begin{array}{l}\text { Afectó las playas de } \\
\text { Cuba Occidental }\end{array}$ \\
\hline
\end{tabular}

Tabla 5B.- Tsunamis en la parte septentrional del Caribe (Lander et al., 2002).

\begin{tabular}{|c|c|c|c|c|}
\hline $\mathbf{N}^{\mathbf{0}}$ & Fecha/Tiempo & Lugar & Nota & $\begin{array}{c}\text { Clasifica- } \\
\text { ción }\end{array}$ \\
\hline 1 & $1688.03 .01 /$ Gregorian & $\begin{array}{c}17.6 \mathrm{~N} / 76.5 \text { O; Jamaica [Port } \\
\text { Royal] }\end{array}$ & & $\mathrm{P}$ \\
\hline 2 & 1690.04 .16 & $\begin{array}{c}17.5 \mathrm{~N} / 61.5 \mathrm{O} \text {; Ms=8.0; U.S. Islas } \\
\text { Virgenes }\end{array}$ & $\mathrm{V}$ \\
\hline 3 & $1692.06 .07 / 11: 43 \mathrm{LT}$ & $\begin{array}{c}17.8 \mathrm{~N} / 76.7 \text { O; Ms=7.7; Jamaica } \\
\text { [Port Royal, Liganee (Kingston). } \\
\text { Saint Ann's Bay] }\end{array}$ & $\begin{array}{c}\text { Runup=1.8m; } \\
2000 \text { muertos }\end{array}$ & $\mathrm{V}$ \\
\hline 4 & $1751.09 .15 / 19: 00 \mathrm{UT}$ & $\begin{array}{c}18.5 \mathrm{~N} / 70.7 \text { O; Ms=7.3; La } \\
\text { Española [Haiti] }\end{array}$ & $\mathrm{P}$ \\
\hline 5 & 1751.11 .21 & $\begin{array}{c}18.3 \mathrm{~N} / 72.3 \text { O; Haiti [Port-au- } \\
\text { Prince] }\end{array}$ & & $\mathrm{P}$ \\
\hline 6 & $1751.10 .18 / 19: 00 \mathrm{UT}$ & $\begin{array}{c}18.5 \mathrm{~N} / 70.7 \text { O; Ms=7.3; La } \\
\text { Española [Azua de Compostela, } \\
\text { Santo Domingo. Santa Cruz, El } \\
\text { Seíbo] }\end{array}$ & & $\mathrm{V}$ \\
\hline
\end{tabular}




\begin{tabular}{|c|c|c|c|c|}
\hline $\mathbf{N}^{0}$ & Fecha/Tiempo & Lugar & Nota & $\begin{array}{l}\text { Clasifica- } \\
\text { ción }\end{array}$ \\
\hline 7 & 1755.11.01/09:50 LT & Lisboa & $\begin{array}{l}\text { Cuba [Santia- } \\
\text { go de Cuba] }\end{array}$ & $\mathrm{V}$ \\
\hline 8 & 1766.06.12/04:45 UT & $\begin{array}{l}20.0 \mathrm{~N} / 75.5 \mathrm{O} \text {; Cuba [Santiago de } \\
\text { Cuba. Bayamo], Jamaica }\end{array}$ & & $\mathrm{P}$ \\
\hline 9 & 1769 & $\begin{array}{l}18.5 \quad \mathrm{~N} / \\
\text { Prince] }\end{array}$ & & $\mathrm{P}$ \\
\hline 10 & 1770.06.03/19:15 LT & $\begin{array}{l}\text { Haiti [Golfo de la } \text { Gonave } \\
\text { Arcahaie] }\end{array}$ & & $\mathrm{V}$ \\
\hline 11 & 1775.02 .11 & $\begin{array}{l}19.0 \mathrm{~N} / 72.4 \mathrm{O} \text { or } 20.0 \mathrm{~N} / 15.8 \mathrm{O} ; \\
\text { La Española, Cuba }\end{array}$ & & $\mathrm{P}$ \\
\hline 12 & 1775.03 & $\begin{array}{l}19.0 \mathrm{~N} / 72.3 \mathrm{O} \text { or } 20.0 \mathrm{~N} / 15.8 \mathrm{O} ; \\
\text { La Española }\end{array}$ & & $\mathrm{P}$ \\
\hline 13 & 1775.12 .18 & $\begin{array}{l}19.2 \text { N/ } 70.3 \text { O; MMI= VIII; La } \\
\text { Española, Cuba }\end{array}$ & & $\mathrm{P}$ \\
\hline 14 & 1780.10.03/22:00 LT & $\begin{array}{l}\text { 18.1 N/78.1 O; Jamaica [Savana La } \\
\text { Mar] }\end{array}$ & $\begin{array}{l}\text { Run-up }=3.0 \mathrm{~m} ; \\
10 \text { muertos }\end{array}$ & $\mathrm{P}$ \\
\hline 15 & 1781.08 .01 & $\begin{array}{l}18.2 \mathrm{~N} / 78.1 \mathrm{O} ; \text { Jamaica [Montego } \\
\text { Bay] }\end{array}$ & & $\mathrm{P}$ \\
\hline 16 & 1787.10.27/14:20 LT & $\begin{array}{l}18.4 \text { N/ } 77.9 \text { O; Jamaica [Montego } \\
\text { Bay] }\end{array}$ & & $\mathrm{P}$ \\
\hline 17 & 1812.11.11/10:50 UT & $\begin{array}{l}18.0 \mathrm{~N} / 76.5 \mathrm{O} \text {; Jamaica [Annotto } \\
\text { Bay] }\end{array}$ & & $\mathrm{P}$ \\
\hline 18 & 1842.05.07/17:30 LT & $\begin{array}{l}19.7 \mathrm{~N} / 72.8 \mathrm{O} ; \mathrm{Ms}=7.7 \text {; Haiti } \\
\text { [Mole St. Nicolas. Cap Haitien, } \\
\text { Port-de-paix. Forte-Liberte], } \\
\text { República Dominicana [Santiago de } \\
\text { los Caballeros, Santo Domingo, } \\
\text { Costa Norte de La Española] }\end{array}$ & $\begin{array}{l}\text { Run-up }=5.0 \mathrm{~m} ; \\
\sim 5,000 \\
\text { muertos }\end{array}$ & V \\
\hline 19 & $1852.07 .17 / 07: 25$ & $\begin{array}{l}20.0 \mathrm{~N} / 75.8 \mathrm{O} ; \text { Cuba [Santiago de } \\
\text { Cuba] }\end{array}$ & & $\mathrm{P}$ \\
\hline 20 & 1860.03 .08 & $\begin{array}{l}19.0 \mathrm{~N} / 72.0 \mathrm{O} \text {; La Española [Golfo } \\
\text { de la Gonave, Les Cayes, Acquin, } \\
\text { Anse-a-Veau] }\end{array}$ & & V \\
\hline 21 & $1867.11 .18 / 18: 45$ & $\begin{array}{l}18.0 \mathrm{~N} / \quad 65.5 \quad \mathrm{O} ; \mathrm{Ms}=7.5 ; \text { Islas } \\
\text { Vírgenes }[\text { [St. Croix y St. Thomas] }\end{array}$ & $\begin{array}{l}\text { Run- } \\
\text { up=19.8m; } \\
\mathrm{M}_{\mathrm{T}}=2-3\end{array}$ & V \\
\hline 22 & 1874.03.11/04:30 LT & $18.3 \mathrm{~N} / 64.9 \mathrm{O}$; Antillas Menores & Islas Vírgenes & $\mathrm{P}$ \\
\hline 23 & 1881.08 .12 & 19.9 N/ 76.8 O; Jamaica [Kingston] & $\begin{array}{l}\text { Run- } \\
\text { up }=0.46 \mathrm{~m}\end{array}$ & $\mathrm{P}$ \\
\hline 24 & 1883.08.27/10:00 LT & $\begin{array}{l}05.8 \text { S. } 106.3 \mathrm{E} \text {; Indonesia (Kraka- } \\
\text { toa Volcano) }\end{array}$ & $\begin{array}{l}\text { Islas Vírgenes } \\
\text { (St. Thomas) }\end{array}$ & $\mathrm{V}$ \\
\hline 25 & 1887.09.23/12:00 UT & $\begin{array}{l}19.7 \text { N / 74.4 W; Haiti [Mole Saint- } \\
\text { Nicolas, Jeremie, Anse-d'Hainault, } \\
\text { Point Tiburón] }\end{array}$ & & $\mathrm{V}$ \\
\hline
\end{tabular}




\begin{tabular}{|c|c|c|c|c|}
\hline $\mathbf{N}^{\mathbf{o}}$ & Fecha/Tiempo & Lugar & Nota & $\begin{array}{l}\text { Clasifica- } \\
\text { ción }\end{array}$ \\
\hline 26 & 1907.01.14/21:36 UT & $\begin{array}{l}18.1 \mathrm{~N} / 76.7 \mathrm{~W} \text {; Ms=6.5; Jamaica } \\
\text { [Hope Bay, Orange Bay, Sheerness } \\
\text { Bay, St. Ann's Bay, Annotto Bay, } \\
\text { Port Maria, Ocho Rios, Bluff Bay, } \\
\text { Port Antonia, Kingston] }\end{array}$ & Run-up $=2.5 \mathrm{~m}$ & $\mathrm{~V}$ \\
\hline 27 & 1918.10.11/04:14 UT & $\begin{array}{l}18.5 \mathrm{~N} / 67.5 \mathrm{~W} \text {; Ms=7.5; Puerto } \\
\text { Rico [Aguadilla. Punta Agujereada; } \\
\text { Punta Higuero, Punta Borinquen, } \\
\text { Isla Mona, Rio Culebrinas, Bahia } \\
\text { de Boqueron, Isabella, Cayo } \\
\text { Cardona, Guanica, Mayaguez, Isla } \\
\text { Caja de Muertos, Puerto Arecibo, } \\
\text { Rio Grande, Rio Grande de Loiza, } \\
\text { Playa Ponce], República Dominica- } \\
\text { na [Santo Domingo (Rio Ozama)] }\end{array}$ & $\begin{array}{l}\text { Run-up=6.1 } \\
\text { m; } \quad 140 \\
\text { muertos }\end{array}$ & $\mathrm{V}$ \\
\hline 28 & 1918.10.24/03:43 UT & 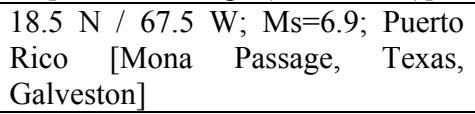 & & $\mathrm{V}$ \\
\hline 29 & 1931.10 .01 & $\begin{array}{l}21.5 \mathrm{~N} / 80.0 \mathrm{~W} ; \\
\text { Panchita, Raba [Playa } \\
\text { Villas] }\end{array}$ & & $P$ \\
\hline 30 & 1932.02.03/06:16 UT & $\begin{array}{l}19.5 \mathrm{~N} / 75.6 \mathrm{~W} ; \mathrm{Ms}=6.8 ; \text { Cuba } \\
\text { [Santiago de Cuba] }\end{array}$ & & $P$ \\
\hline 31 & 1939.08.15/03:52 UT & $\begin{array}{l}22.5 \mathrm{~N} / 79.2 \mathrm{~W} ; \mathrm{Ms}=8.1 ; \text { Cuba } \\
\text { [Cayo Francés] }\end{array}$ & & $\mathrm{V}$ \\
\hline 32 & 1946.08.04/17:51 UT & $\begin{array}{l}\text { 19.3 N / } 68.9 \text { W; Ms=8.1; Repúbli- } \\
\text { ca Dominicana [Matancitas, Julia } \\
\text { Molina, Cabo Samana], Haiti y } \\
\text { Puerto Rico [San Juan] }\end{array}$ & $\begin{array}{l}\text { Run-up }=5.0 \\
\text { m; } \quad 1,790 \\
\text { muertos }\end{array}$ & $\mathrm{V}$ \\
\hline 33 & 1946.08.08/13:28 UT & $\begin{array}{l}19.5 \mathrm{~N} / 69.5 \mathrm{~W} ; \mathrm{Ms}=7.9 ; \text { Puerto } \\
\text { Rico [Aguadilla, Mayagüez, San } \\
\text { Juan] }\end{array}$ & 75 muertos & $\mathrm{V}$ \\
\hline 34 & 1953.05.31/19:58 UT & $\begin{array}{l}19.7 \text { N / } 70.7 \text { W; República Domi- } \\
\text { nicana [Puerto Plata] }\end{array}$ & $\begin{array}{l}\text { Run-up }=0.06 \\
\mathrm{~m}\end{array}$ & $P$ \\
\hline 35 & 1989.11.01/10:25 UT & $\begin{array}{l}19.0 \mathrm{~N} / 68.8 \mathrm{~W} ; \mathrm{Ms}=5.2 ; \text { Puerto } \\
\text { Rico [Cabo Rojo, E Nuevo Dia] }\end{array}$ & & $\mathrm{V}$ \\
\hline 36 & 1991.04.22/21:56 UT & $\begin{array}{l}09.7 \mathrm{~N} / 83.1 \mathrm{~W} ; \mathrm{Ms}=7.4 ; \text { Costa } \\
\text { Rica }\end{array}$ & Islas Vírgenes & $\mathrm{V}$ \\
\hline
\end{tabular}

NOTA: $\mathrm{P}=$ Probable, $\mathrm{V}=$ Verificado.

Tabla 6.- Tsunamis en el Caribe desde fuentes lejanas.

\begin{tabular}{|l|c|}
\hline \multicolumn{1}{|c|}{ Región fuente } & Total \\
\hline Suroeste de la Península Ibérica (Portugal) & 2 \\
\hline Indonesia (volcán Krakatoa) & 1 \\
\hline Total & $\mathbf{3}$ \\
\hline
\end{tabular}


Tabla 7A.- Tsunamis in Venezuela (Shubert, 1994).

\begin{tabular}{|c|c|c|c|c|}
\hline $\mathbf{N}^{\mathbf{0}}$ & Fecha / Tiempo & Localidad & $\mathbf{M}_{\mathbf{T}} / \mathbf{I}$ (MM) & Nota \\
\hline 1 & $1530.09 .01 / 14: 30$ UT & Cumaná & $-/ \mathrm{X}$ & \\
\hline 2 & 1900.10 .29 & Cumaná & $8.4 /-$ & \\
\hline 3 & 1906.01 .31 & Cumaná & $8.9 /-$ & No Lander \\
\hline 4 & 1929.01 .17 & Cumaná & $8.1 /-$ & Ms=6.9; $11: 52 \mathrm{UT}$ \\
\hline 5 & 1868.08 .13 & Río Caribe & $8.5 /-$ & No Lander \\
\hline
\end{tabular}

Nota: $\mathrm{M}_{\mathrm{T}}=$ Magnitud de tsunamis; $\mathrm{I}=$ Intensidad Sísmica (Mercalli Modificada); Lander= Lander et al., 2002.

Tabla 7B.- Tsunamis in Venezuela (Lander et al., 2002).

\begin{tabular}{|c|c|c|c|}
\hline $\mathbf{N}^{\mathbf{0}}$ & Fecha / Tiempo & Localidad & M / I (MM) \\
\hline 1 & $1530.09 .01 / 14: 30 \mathrm{UT}$ & Cumaná & $-/$ X \\
\hline 2 & 1853.07 .15 & Cumaná & $6.7 /-$ \\
\hline 3 & 1900.10 .29 & Cumaná & $8.4 /-$ \\
\hline 4 & $1906.01 .31 / 15: 36$ & Cumaná & $6.9 /-$ \\
\hline 5 & 1929.01 .17 & Cumaná & $7,0 /-$ \\
\hline 6 & $1997.07 .09 / 19: 24$ UT & Isla Margarita & \\
\hline 7 & 1802.05 .05 & Río Orinoco & \\
\hline
\end{tabular}

\subsection{Principales características de la zona septentrional del Caribe}

Álvarez et al. (1999); Cotilla (2003, 1998); Cotilla y Álvarez (1998); Cotilla y Córdoba (2010, 2010A, 2010B); Montelieu (1988, 1933); Morales y Pedroso (1931); y Rodríguez Ferrer (1876) recogen las características de la sismicidad de la parte septentrional del Caribe y de Cuba en particular.

Los terremotos más fuertes de la zona septentrional caribeña al este de Jamaica y Cuba están en la figura 5. Mientras que una selección de los mecanismos focales de la zona septentrional del Caribe aparece en la figura 6. De esta última es factible comprobar que los mecanismos de tipo normal e inverso predominan en las inmediaciones de Islas Vírgenes, Puerto Rico, La Española, y Jamaica. Esas son también las zonas que han experimentado en el tiempo los terremotos más fuertes de la región.

A las Islas Vírgenes, Puerto Rico, Jamaica, y La Española han sido asociados algunos terremotos fuertes y tsunamis (Tabla 5A y 5B). En estos casos no ha existido vínculo alguno con volcán. Se ha demostrado que algunos de esos tsunamis han afectado a los territorios isleños vecinos. Pero siempre los mayores daños fueron locales.

\subsection{Información de Cuba}

Para la configuración de este epígrafe se han empleado los siguientes trabajos: Álvarez et al., 1999; Chuy, 1999; Chuy y Pino, 1982; Chuy et al., 1980; Cotilla, 2003; Cotilla y Córdoba, 2010, 2010A, 2010B. Sobre esa base es factible sostener que Cuba es una estructura emergida del borde sur de la placa de Norteamérica, y que ha sufrido algunos eventos sísmicos fuertes (Tabla 9). Esos eventos no han 
producido tsunamis y puede explicarse por el tipo de fallas sismoactivas. Sin embargo, la parte suroriental [bahía de Santiago de Cuba] ha sido afectada ligeramente, al menos, por un tsunami de una fuente lejana [Portugal] el 01.11.1755.

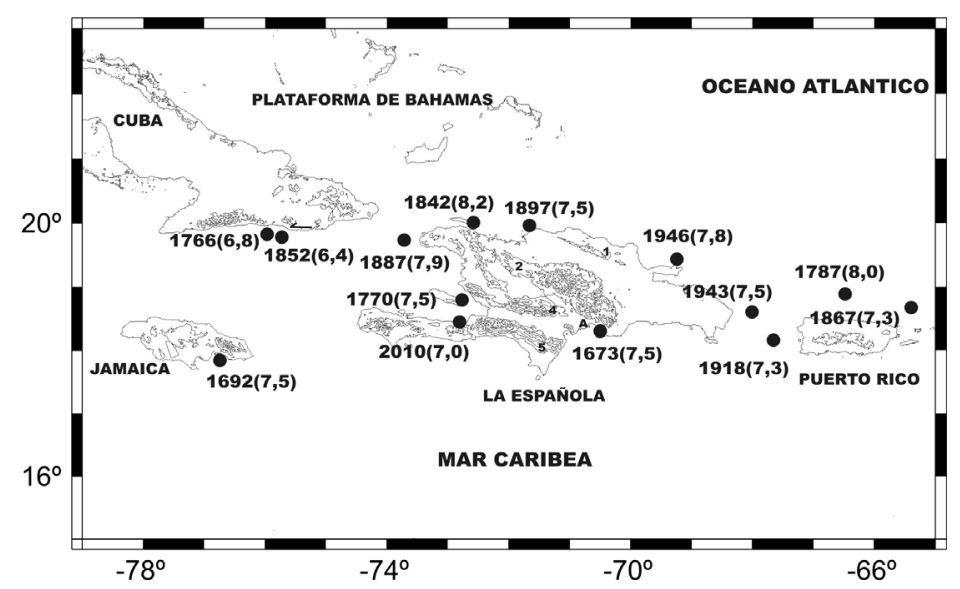

Figura 5.- Terremotos Fuertes del nordeste del Caribe. [Circulo negro= epicentro; $1766(7,5)=$ año (magnitud). $]$

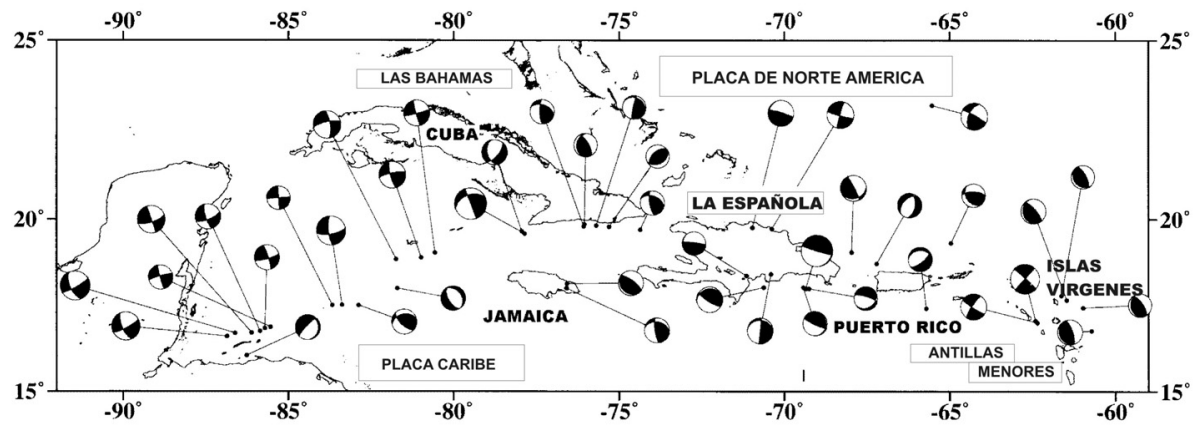

Figura 6.- Selección de mecanismos focales del Caribe septentrional.

De acuerdo con algunos autores en Cuba se han determinado, hasta el momento, como máximo siete tsunamis (Tabla 10). De la tabla anterior se aprecia que hay una diferencia en tres eventos atendiendo a las dos fuentes bibliográficas. El autor también ha expuesto su desacuerdo con algunos de esos datos (Cotilla, 2007). Así, las investigaciones en los fondos documentales del Archivo de Indias en Sevilla para los dos terremotos más fuertes ocurridos en el entorno de Santiago de Cuba [11.06.1766 y 20.08.1852] (Figura 5B) no contienen mención alguna a movimientos de masas de agua en la zona costera (Cotilla, 2003; Cotilla y Córdoba, 2010A). En ese mismo entorno está el terremoto del 03.02.1932 y que Montelieu (1933) 
estudió desde el primer momento sobre el terreno y descartó el tsunami (Tabla 11B). Todo esto se puede explicar a partir del hecho concreto del mecanismo focal predominante en el segmento de la falla Oriente (Figura 6). Hay otros dos eventos asociados a terremotos, pero en la parte central y norte de Cuba, 01.10.1931 y 04.08.1939 (Figuras 3 y 4), que se han documentado con penetraciones del mar en la isla (Tabla 11B). El autor sólo considera cierto al evento de 1931 y le vincula con la falla Nortecubana, de tipo vertical-normal (Cotilla, 2007). Esa falla ha sido responsable de otros eventos sísmicos, el más importante de magnitud 6.2 [28.02.1914 / 05:19:00 UT, Gibara, Holguín] (Figuras 2B, 3 y 4). Es decir, esa falla no ha tenido una magnitud asociada de 8.1 como se sostiene por Lander et al. (2002). Y con respecto al evento de 1946 [de República Dominicana] indicamos que éste tiene un matiz de mucha connotación y que al parecer ha pasado inadvertido para algunos especialistas. Ese matiz es que las playas de Cuba Occidental mencionadas por Rubio (1982) (Figura 7), están situadas algunos kilómetros [ 300] más al oeste que la región centro norte [Remedios, Caibarién, etc.] y que ellas aparecen en la parte suroeste del cambio de orientación de la estructura de la isla de Cuba al SO (Figura 3). Observar que, atendiendo a las fuentes bibliográficas empleadas, la región de Remedios-Caibarién no fue afectada por dicho tsunami aunque ella enfrenta con dirección NW en la parte oriental del arco [cóncavo al norte] cubano (Figura 2B). Esto físicamente no tiene sentido.

Tabla 8.- Fallecidos por tsunamis en el Caribe de acuerdo con distintos autores.

\begin{tabular}{|c|c|c|c|}
\hline $\mathbf{N}^{\mathbf{0}}$ & Referencia & Lugar & $\begin{array}{c}\text { Cantidad de } \\
\text { fallecidos }\end{array}$ \\
\hline 1 & McNamara et al., 2005 & Caribe & 9,600 \\
\hline 2 & Lander et al., 2002 & Caribe septentrional & $\sim 9,000$ \\
\hline 3.1 & Hillebrant, 2005 & República Dominicana - 1946 & 1,800 \\
\hline 3.2 & Lander et al., 2002 & & 1,790 \\
\hline 3.3 & Rubio, 1982 & 75 \\
\hline 4.1 & Lander et al., 2002 & Puerto Rico - 1918 & 140 \\
\hline 4.2 & Nealon y Dillon, 2001 & & 91 \\
\hline 4.3 & Mercado y McCann, 1998 & & 40 \\
\hline 4.4 & Rubio, 1982 & & 8 \\
\hline 4.5 & Robson, 1964 & & 30 \\
\hline 5 & Fernández et al., 2000 & Panamá - 1882 & 190 \\
\hline
\end{tabular}

\begin{tabular}{|c|c|c|c|}
\hline $\mathbf{N}^{\mathbf{0}}$ & Referencia & Lugar & $\begin{array}{c}\text { Cantidad de } \\
\text { fallecidos }\end{array}$ \\
\hline 6 & $\begin{array}{c}\text { Hillebrant y Huérfano, } \\
2004\end{array}$ & Islas Vírgenes -1867 & 17 \\
\hline 7.1 & Lander et al., 2002 & Haití -1842 & 500 \\
\hline 7.2 & Lander y Whiteside, 1997 & & $200-300$ \\
\hline 7.3 & Rubio, 1982 & & 200 \\
\hline 8.1 & Lander et al., 2002 & Jamaica -1780 & 10 \\
\hline
\end{tabular}




\begin{tabular}{|c|c|c|c|}
\hline 8.2 & Rubio, 1982 & & 300 \\
\hline 9 & Rubio, 1982 & Haití -1770 & 200 \\
\hline 10.1 & Lander et al., 2002 & Jamaica -1692 & 2,000 \\
\hline 10.2 & Rubio, 1982 & & 2,000 \\
\hline
\end{tabular}

Tabla 9.- Terremotos fuertes de Cuba Oriental.

\begin{tabular}{|c|c|c|c|c|}
\hline $\mathbf{N}^{\mathbf{0}}$ & Fecha / Tiempo & Coordenadas & M / I (MSK) & Localidad \\
\hline 1 & $1766.06 .11 / 00: 00$ & $19.9 \mathrm{~N} / 76.1 \mathrm{O}$ & $6.8 / 9$ & S.Cuba \\
\hline 2 & $1800.10 .14 / 05-06$ & $19.9 \mathrm{~N} / 75.9 \mathrm{O}$ & $6.4 / 8$ & S.Cuba \\
\hline 3 & $1826.09 .18 / 09: 29$ & $19.75 \mathrm{~N} / 75.35 \mathrm{O}$ & $6.4 / 8-9$ & S.Cuba \\
\hline 4 & $1842.07 .07 / 17: 25$ & $19.75 \mathrm{~N} / 75.35 \mathrm{O}$ & $6.8 / 8$ & S.Cuba \\
\hline 5 & $1852.08 .20 / 14: 05$ & $19.75 \mathrm{~N} / 75.32 \mathrm{O}$ & $6.4 / 8$ & S.Cuba \\
\hline 6 & $1932.02 .03 / 12: 35$ & $19.75 \mathrm{~N} / 75.32 \mathrm{O}$ & $6.75 / 8$ & S.Cuba \\
\hline
\end{tabular}

Tabla 10.- Tsunamis en Cuba de acuerdo con dos autores diferentes.

\begin{tabular}{|c|c|l|c|c|l|}
\hline $\mathbf{N}^{\mathbf{0}}$ & Fecha & \multicolumn{1}{|c|}{ Lugar } & Rubio & $\begin{array}{c}\text { Lan- } \\
\text { der }\end{array}$ & \multicolumn{1}{|c|}{ Nota } \\
\hline 1 & 1755.11 .01 & Santiago de Cuba & $\mathrm{X}$ & $\mathrm{X}$ & Teletsunami \\
\hline 2 & 1766.06 .12 & Santiago de Cuba & & $\mathrm{X}$ & $\begin{array}{l}\text { Cotilla, 2003 [no } \\
\text { coincide] }\end{array}$ \\
\hline 3 & 1775.12 .18 & Santiago de Cuba & $\mathrm{X}$ & $\mathrm{X}$ & \\
\hline 4 & 1852.07 .17 & Santiago de Cuba & $\mathrm{X}$ & $\mathrm{X}$ & $\begin{array}{l}\text { Cotilla, 2010A [no } \\
\text { coincide] }\end{array}$ \\
\hline 5 & 1931.10 .01 & $\begin{array}{l}\text { Playa Panchita- } \\
\text { Rancho Veloz }\end{array}$ & & $\mathrm{X}$ & $\mathrm{X}$ \\
\hline 6 & 1932.02 .03 & Santiago de Cuba & $\mathrm{X}$ & $\begin{array}{l}\text { Montelieu, 1933 } \\
\text { [no coincide] }\end{array}$ \\
\hline 7 & 1939.08 .04 & $\begin{array}{l}\text { Cayo Francés } \\
\text { Cotilla, 2007 [no } \\
\text { coincide por la } \\
\text { magnitud] }\end{array}$ \\
\hline 8 & 1946.08 .08 & $\begin{array}{l}\text { Playas de Cuba } \\
\text { Occidental }\end{array}$ & & & $\begin{array}{l}\text { Cotilla, 2007 [no } \\
\text { coincide] }\end{array}$ \\
\hline
\end{tabular}

Nota: Rubio= Rubio (1982); Lander= Lander et al., 2002.

Como se aprecia en las tablas 3A, 3B, 4A, 4B, 7A, y 7B no ha habido influencias de tsunamis por fuentes caribeñas en Cuba. De hecho, Rubio (1982) aseguró que Cuba no requiere de estudios ni medidas especiales en el tema de tsunamis. Sin embargo, en las tablas 5A y 11B hay datos de la influencia de otras fuentes regionales. Esos son los casos de Haití. Esas dos tablas permiten descartar a Jamaica. Y el autor pone en duda la influencia de la República Dominicana, como expuso anteriormente. En el otro sentido, se ha comprobado que los tsunamis ocurridos en Cuba [locales], en particular los de la zona septentrional, no han influido en territorios aledaños. Ellos siempre fueron de baja magnitud y muy localizados. 
La modelización realizada para el noreste caribeño del evento de 1918 (Mercado y McCann, 1998) supone la influencia del tsunami en Cuba; sin embargo, ese acontecimiento realmente no afectó Cuba. En igual sentido sucede con los eventos del oeste, del suroeste, y del sur del Caribe. Ya estaba descartado el Golfo de México. Y los eventos tsunamigénicos de Jamaica, República Dominicana, e Islas Vírgenes tampoco se han percibido en Cuba. Sin embargo, una fuente lejana como el suroeste de la Península Ibérica si ha producido oscilaciones en Santiago de Cuba 78 horas después del terremoto principal.

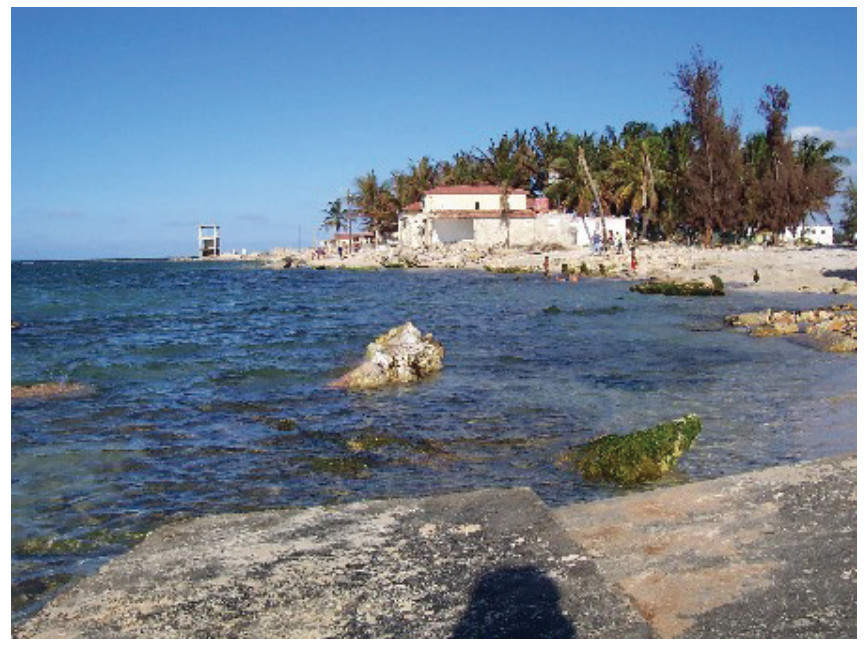

Figura 7.- Imagen de la playa Santa Fé, La Habana.

Los tsunamis del 1867.11.18 en Islas Vírgenes [Ms=7,5; $18.0 \mathrm{~N} / 65.5 \mathrm{O}]$ y del 1918.10.11 en Puerto Rico [Ms=7,5; 18.5 N / 67.5 W] que tuvieron como fuentes terremotos, muestran una contradicción en cuanto a las afectaciones por oleaje en Cuba. Así, el primero se hizo sentir en toda la región oriental cubana, mientras que el segundo, a pesar de estar más cerca $\left[2^{\circ}\right]$ que el primero, no produjo afectaciones. ¿Por qué? También hay una paradoja, no indicada con anterioridad, que concierne a los eventos tsunamigénicos de 1867 [Islas Vírgenes] y 1918 [Puerto Rico]. Ella consiste en que esos y otros territorios del Caribe, incluida la parte suroriental de Cuba, fueron afectados, al menos por el evento lejano de 1755 y esos dos eventos no afectaron a la Península Ibérica. Es decir, que hay, a juicio del autor: 1) una muy diferente potencialidad de las fuentes; 2) un evidente problema de direccionalidad de la energía. Estos aspectos consideramos deben ser abordados en algún momento.

Las tablas 11A y 11B contienen las informaciones detalladas de los tsunamis ocurridos en Cuba. En ellas se aprecian diferencias y matices de interés entre las anotaciones de los distintos autores. Todo esto entendemos hay que revisarlo. 
Tabla 11A.- Terremotos con tsunamis en Cuba.

\begin{tabular}{|c|c|c|c|c|}
\hline $\mathbf{N}^{\mathbf{0}}$ & Fecha/Tiempo & Coordenadas & $\mathbf{M} / \mathbf{I} / \mathbf{h}(\mathbf{k m})$ & Fuente \\
\hline 1 & $1755.11 .01 /$ & & & MP \\
\hline \multirow[t]{3}{*}{2} & 1766.06.12/05:14UT & $19.80 \mathrm{~N} 76.10^{\circ}$ & $7.6 / \mathrm{IX} / 35$ & $\mathrm{Ch}$ \\
\hline & 1766.06.11/00:00LT & $19.9 \mathrm{~N} 76.1^{\circ}$ & $6.8 / \mathrm{IX} / 25$ & $\mathrm{~A}$ \\
\hline & 1766.06.11/00:00LT & $19.9 \mathrm{~N} 76.1^{\circ}$ & $6.8 / \mathrm{IX} / 25$ & $\mathrm{C}$ \\
\hline 3 & $1775.12 .18 /$ & & & \\
\hline 4.1 & 1852.07.17/14:05UT & $19.77 \mathrm{~N} 75.35^{\circ}$ & $7.3 / \mathrm{IX} / 30$ & $\mathrm{Ch}$ \\
\hline \multirow[t]{2}{*}{4.2} & 1852.08.20/14:05UT & $19.75 \mathrm{~N} 75.32^{\circ}$ & $7.3 / \mathrm{IX} / 30$ & A \\
\hline & $1852.08 .20 /$ & & & $\mathrm{CC}$ \\
\hline \multirow[t]{2}{*}{5} & $1931.10 .01 /$ & & & MP \\
\hline & $1931.10 .01 /$ & & & Ch3 \\
\hline \multirow[t]{2}{*}{6} & $1932.02 .03 /$ & & & $\mathrm{M}$ \\
\hline & 1932.02.03/06:15UT & $19.80 \mathrm{~N} 75.80^{\circ}$ & 6,75/VIII/- & Ch2 \\
\hline 7 & $1939.08 .04 / 23: 55 \mathrm{LT}$ & $22^{\circ} 30^{\prime} \mathrm{N} 79^{\circ} 15^{\prime} \mathrm{O}$ & $5.3-5.9 / \mathrm{VII} /-$ & Ch3 \\
\hline
\end{tabular}

Nota: Fuentes: $\mathrm{A}=$ Álvarez et al., 1999; $\mathrm{C}=$ Cotilla, 2003; $\mathrm{CC}=$ Cotilla y Córdoba, 2010A; $\mathrm{Ch}=$ Chuy, 1999; Ch1 = Chuy y Pino, 1982; Ch2= Chuy T., González B. y Escalona S., 1980; $\mathrm{L}=$ Lander et al., 2002; M= Montelieu, 1933; MP= Morales y Pedroso, 1931; I= Intensidad sísmica (escala MSK).

\section{Apuntes finales y conclusiones}

Es posible apreciar en la tabla 1 que no hay tsunamis fuertes en el área Caribe. Esto se explica por su pertenencia a la cuenca del Atlántico. Sin embargo, en el Caribe son frecuentes los ciclones y huracanes (Rodríguez, 1989). Estos muy dañinos fenómenos de desarrollo vertical son conocidos por todos los residentes en dicha región. Ellos son responsables de las muy elevadas pérdidas económicas, así como de las numerosas pérdidas humanas. Dichos organismos ocurren preferentemente en el intervalo temporal de septiembre - noviembre y provocan la penetración del mar en las zonas costeras de los países que afectan. De hecho hay en general programas de alerta y prevención, de distinto calado e importancia, desde el siglo XIX. El trazado de los recorridos de estos fenómenos es, por lo general, del este al oeste y luego hay un giro al norte, hacia el continente (Figura 2B). Esto fue propuesto y explicitado por el jesuita Benito Viñes Martorell (Cotilla, 2007).

El Caribe, como bolsa marina en la zona Tropical Atlántica (Alisov, 1989), es estacionalmente afectada por otros procesos meteorológicos activos como los frentes polares o "frentes fríos". Ellos pueden provocar penetraciones del mar en las costas bajas y marejadas muy importantes (Rodríguez, 1983) (Figura8). Esto permite al autor, una vez constatados algunos errores en catálogos y relaciones sobre tsunamis, sostener que los fenómenos meteorológicos mencionados pudieron ser confundidos como tsunamis. 
Tabla 11B.- Descripción de los terremotos con tsunamis en Cuba.

\begin{tabular}{|c|c|}
\hline $\mathbf{N}^{\mathbf{0}}$ & Descripción \\
\hline 1 & $\begin{array}{l}\text { 1755.11.01: Temblor e inundaciones del mar [Santiago de Cuba]. Este terremoto fue } \\
\text { muy desastroso para Lisboa.- Poey, } \mathbf{1 8 5 7} \text {. } \\
\text { 1755: Terremoto y maremoto que inundó la ciudad [Santiago de Cuba].- Miguel } \\
\text { Rodríguez Ferrer, } \mathbf{1 8 7 6} \text {. } \\
\text { 1755.11.01: Santiago de Cuba. Una ola produce daños a las edificaciones de la } \\
\text { bahía.- Rubio, } \mathbf{1 9 8 2} \text {. }\end{array}$ \\
\hline 2 & $\begin{array}{l}\text { 1766.06.01: En el Archivo de Indias (documento 5-Cuba 1051) aparece: “...el movi- } \\
\text { miento de las ondas marinas no eran mayores que } 20 \text { varas como es lo habitual aquí } \\
\text { [bahía de Santiago de Cuba]...”.- Cotilla, 2003. Esto coincide con Rubio, } 1982 .\end{array}$ \\
\hline 3 & 1775.02.11: No hay mención a tsunami.- Chuy y Pino, 1982. \\
\hline 4.1 & $\begin{array}{l}\text { 1852.07.17: Santiago de Cuba. La bahía se vió afectada por un fuerte oleaje que } \\
\text { afectó construcciones portuarias y embarcaciones surtas en el puerto. Debió ser } \\
\text { producto de un sismo que afectó al buque de guerra de los Estados Unidos "Tropic" a } \\
70 \text { millas de Jamaica.- Rubio, } 1982 \text {. }\end{array}$ \\
\hline 4.2 & $\begin{array}{l}\text { 1852.08.20: } 14: 05,19.75 \mathrm{~N} 75.32 \mathrm{~W}, \mathrm{Ms}=6.4, \mathrm{I}(\mathrm{MSK})=8, \mathrm{~h}=30 \mathrm{~km} \text {, no hubo tsunami } \\
\text { en Santiago de Cuba.- Cotilla y Córdoba, 2010A. }\end{array}$ \\
\hline 5 & $\begin{array}{l}\text { 1867.11.18: Islas Vírgenes [St. Thomas]. Olas sísmicas observadas en los puertos de } \\
\text { la provincial de Oriente [Cuba].- Rubio, } \mathbf{1 9 8 2} \text {. }\end{array}$ \\
\hline
\end{tabular}

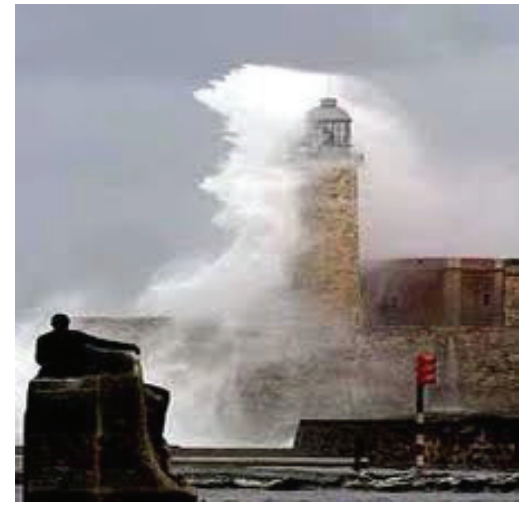

Figura 8.- Imagen del oleaje por un frente "frío" [o norte] en La Habana.

De otra parte, el conocimiento por parte de la población sobre los tsunamis en el Caribe es con diferencia muy bajo en comparación con los ciclones y huracanes. Las cifras sobre las afectaciones de los tsunamis en el Caribe, y en particular la cantidad de muertos, resultan ser diferentes según distintas fuentes. Esto significa que resta un importante trabajo de homogenización y verificación en las fuentes bibliográficas originales.

Por último, hay otra apreciación que no hemos podido explicar, por el momento, a pesar de haber buscado en la documentación presentada. Esto se refiere al 
hecho concreto de las afectaciones en la bahía de Santiago de Cuba (Figura 9) por los tsunamis de Portugal y de Haití. Sin embargo, la bahía de Guantánamo que está situada en la misma latitud y zona costera suroriental, con la misma orientación NE, con una entrada más favorable en cuanto a abertura al Mar Caribe, con un fondo marino mucho menos profundo, y geográficamente más cercana a esas fuentes, que la homologa Santiago de Cuba, no aparece en los datos. ¿Qué ha sucedido? Y con relación a la peligrosidad y el riesgo tsunamigénico en Cuba, sostenemos que con los datos existentes y el enfoque presentado, son muy bajos para fuentes lejanas y regionales, aunque un poco más elevados para fuentes locales de la zona centroseptentrional de la isla.

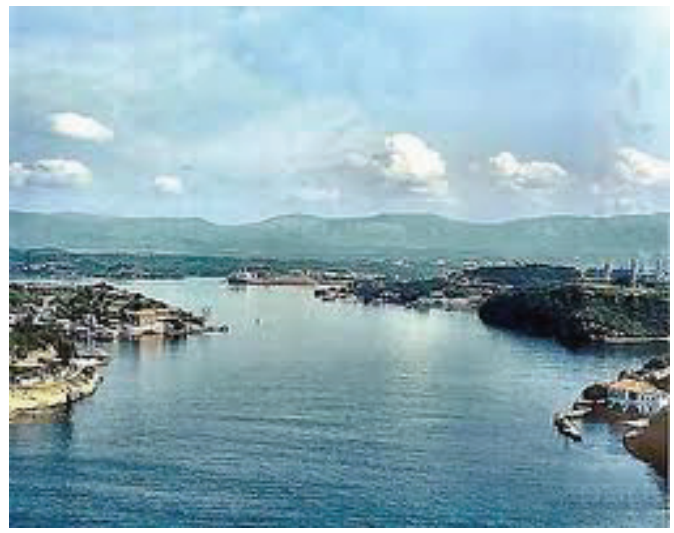

Figura 9.- Imagen desde la boca de la bahía santiagüera hacia el norte.

\section{Agradecimientos}

$\mathrm{La}$ financiación provino fundamentalmente de los siguientes proyectos: TOPOIBERIA (CONSOLIDER 52Q6016), CTM 2006-13666-C02-02, CTM 200802955-E/MAR, GR35/10-A/910549, y 41-SISMO-HAITI. Al departamento de Física de la Tierra, Astronomía y Astrofísica 1, Facultad de Ciencias Físicas, Universidad Complutense de Madrid por facilitar los medios informáticos.

\section{Referencias bibliográficas}

AFFLECK CAPT. On the agitation of the sea at Antigua: 1 November 1755. Royal Society of London, Philosophical Transactions, Abriged, 11:9-10, 1809 (en Inglés).

ALISOV B.P. Zonas climáticas de la Tierra. En: Nuevo Atlas de Cuba, VI, 1.2, 1989.

ÁlVAREZ L., CHUY T. GARCIA T., MORENO B., ÁlVAREZ H., BLANCO M., EXPÓSITO O. GONZÁLEZ O. Y FERNÁNDEZ A.I. An earthquake catalogue of Cuba and neighbouring areas. The Abdus Salam International Centre for Theoretical Physics, Miramare-Trieste. Internal Report IC/IR/99/1, 60 p., 1999 (en Inglés).

AMBRASEYS N. Y ADAMS R., 1996: Large magnitude Central America earthquakes, 1898-1994. Geoph.Jour.Int., 121:545-556 (en Inglés). 
ARCE M.F., MOLINA E., HAVSKOV J. Y ATAKAN K., 1998: Tsunamis in Central America. Tech. Rep., 11:1-12. Reduction of Natural Disasters in Central America. Earthquake Preparerdness and Hazard Mitigation. Inst. Solid Earth Physics, Univ. Bergen, Norway (en Inglés).

BRGM, Service Public du BRGM. Etude et prévention du risque, sismologique aux Petites Antilles, evaluation de l'alèa sismique su làrchipel de la Guadeloupe. Rapport de synthèses, 1990 (en Francés).

BRINK U.TEN, DANFORTH W., POLLONI C., ANDREWS B., LLANES P., SMITH S., PARKER E. Y UOZUMI T., 2004: New seafloor map of the Puerto Rico Trench helps assess earthquakes and tsunami hazards. EOS, Transactions, American Geophysical Union, 85(37): 1 (en Inglés).

BRYANT E. TSUNAMI: The underrated hazard. Cambridge University Press, 2001 (en Inglés).

CAICEDO J.H., MARTINELLI B., MEYER H. Y STEER R. 1996: Efecto de tsunami del Mar Caribe en la costa de Colombia. En: Memorias del Seminario Nacional de Ciencia y Tecnologías del Mar, Bogotá, 1.

CHUY T. Macrosísmica de Cuba y su aplicación en los estimados de peligrosidad y microzonación sísmica. Tésis en opción al grado de doctor en Ciencias Geofísicas. Ministerio de Ciencia, Tecnología y Medio Ambiente de Cuba. Santiago de Cuba, 1999.

CHUY T. Y PINO O., 1982: Datos macrosísmicos de los terremotos en la provincia Santiago de Cuba. Revista Investigaciones Sismológicas en Cuba, 2:46-136.

CHUY T., GONZÁLEZ B. Y ESCALONA S., 1980: Información macrosísmica de las provincias de Villa Clara, Cienfuegos y Sancti Spíritus. Revista Investigaciones Sismológicas en Cuba, 1:33-57.

COTILLA M.O. Un recorrido por la Sismología de Cuba. Editorial Complutense, Madrid, 2007. ISBN 97-8-47-491827-4.

COTILLA M.O., 2003: The Santiago de Cuba earthquake of 11 June 1766: Some new insights. Geofísica Internacional, 42(4):589-602 (en Inglés).

COTILLA M.O., 1998: An overview on the seismicity of Cuba. J. Seismology, 2:323-335 (en Inglés).

COTILlA M.O. Y ÁLVAREZ J.L., 1998: Esquema de regionalización del potencial de amenaza geológica en Cuba. Revista Geofísica, 49:48-85.

COTILLA M. Y CÓRDOBA D., 2011: Análisis morfotectónico de la Isla de Puerto Rico, Caribe. Revista Geofísica, 52:79-126.

COTILLA M. Y CÓRDOBA D., 2010: Notes on three earthquakes (14.10.1800, 18.09.1826, 07.07.1842) in Santiago de Cuba. Russian Geology and Geophysics, 51(2):228-236 (en Inglés).

COTILLA M. Y CÓRDOBA D., 2010A: The August 20, 1852 earthquake in Santiago de Cuba. Russian Geology and Geophysics, 51:1.157-1.166 (en Inglés).

COTILLA M. Y CÓRDOBA D., 2010B: The Bayamo earthquake (Cuba) of 18 October 1551. International Journal of Geosciences, 1:1-13 (en Inglés).

COTILLA M. Y CÓRDOBA D., 2007: Seismicity and seismoactive faults of Cuba. Russian Geology and Geophysics, 48(6):505-522 (en Inglés).

COTILLA M.O., CÓRDOBA D. Y CALZADILLA M., 2007: Morphotectonic study of Hispaniola. Geotectonics, 41(5):368-391 (en Inglés). 
COTILlA M. Y UDÍAS A., 1999: Geodinámica del límite Caribe-Norteamérica. Rev.Soc.Geol. de España, 12(2):175-186.

COTILlA M., BANKWITZ P., FRANZKE H.J., ÁlVAREZ L., GONZÁlEZ E., DÍAZ J.L., GRUNTHAL G., PILARSKI J. Y ARTEAGA F., 1991: Mapa sismotectónico de Cuba, escala 1:1,000,000. Comunicaciones Cientificas sobre Geofisica y Astronomía, $23,35 \mathrm{p}$.

COTILLA M.O., RUBIO M., ALVAREZ L. Y GRÜNTHAL G., 1997: Potenciales sísmicos sector Centro - Occidental del arco de Las Antillas Mayores. Revista Geofísica, 46:127-150.

CRUZ G. Y WYSS D.S., 1983: Large earthquakes, mean sea level, and tsunamis along the Pacific Coast of Mexico and Central America. Bull.Seismol.Soc.Amer., 73:553-570 (en Inglés).

FERNÁNDEZ M., HAVSKOV J. Y ATAKAN K., 1999: Destructive tsunamis and tsunami warning in Central America. Science of Tsunami Hazards, 17(3):173-186 (en Inglés).

FERNÁNDEZ M., MOLINA E., HAVSKOV J. Y ATAKAN K., 2000: Tsunamis and tsunami hazards in Central America. Natural Hazards, 22:91-116 (en Inglés).

GRASES J. Terremotos destructores del Caribe, 1502-1990. UNESCO-RELACIS, Caracas, 1990.

GRASES J. Relación cronológica de sismos destructores de América Central, lapso 15261900. Vol. 2, UCV, Caracas, 1974.

GRINDLAY N.R., HEARNE M. Y MANN P., 2005: High risk of tsunami in the northern Caribbean. EOS, Trans.Amer. Geophys. Union, 86(12):121-126 (en Inglés).

GUTENBERG B., 1939: Tsunamis and earthquakes. Seism.Soc.Am.Bull., 29:517-526 (en Inglés).

HEILPIN A. Mont Pelee and the tragedy of Martinique. J.B. Lippincott Co., Philadelphia and London, 333 p., 1903 (en Inglés).

HILLEBRANDT-ANDRADE C.G. von. Seismic monitoring in northeastern Caribbean. En: The $17^{\text {th }}$ Caribbean Geological Conference, San Juan Puerto Rico. July 19-21, 2005 (en Inglés).

HILLEBRANDT-ANDRADE C.G. VON Y HUÉRFANO MORENO V. Emergent tsunami warnig system for Puerto Rico and the Virgin Islands. En: Proceedings of the Caribbean Tsunami Workshop, NSF, PR Emergency Management, Sea Grant and the UPR, 15p., 2004 (en Inglés).

HTDB/PAC. Historical tsunami database for the Pacific, 47BC - 2000 AD. CdRoom, Tsunami Laboratory, ICMMG SD RAS, Novosibirsk, Russia, 2001 (en Inglés).

International Tsunami Information Center (ITIC), 2004: Tsunami glossary. [http://www.shoa.cl/oceano/itic/pdf-docs/glossary.html].

JOHNSTON S.T. Y THORKELSON D.J., 1997: Cocos-Nazca slab window beneath Central America. Earth Planet.Sci. Letters, 146:465-474 (en Inglés).

KELLOG J.N. Y BONINI W.E., 1982: Subduction of the Caribbean plate and basement uplifts in the overriding South America Plate. Tectonics, 1:251-276 (en Inglés).

KUROIWA J. Investigation of tsunami hazard mitigation in developing countries. Seminar Workshop on Technical Aspects of Tsunami, Analysis, Prediction and Communication. Sidney, British Columbia, Canada, 1985 (en Inglés).

LANDER J.F. Y WHITESIDE L.S., 1997: Caribbean tsunamis: an initial history [http://rmoclis.upr.clu.edu/tsunamis/Lander/J\_Lander.html] (en Inglés). 
LANDER J.F., WHITESIDE L.S. Y LOCKRIDGE P.A., 2002: A brief history of tsunamis in the Caribbean Sea. Science of Tsunami Hazards, 20(2):57-94 (en Inglés).

LYNCH J.J. Y BODLE R.R., 1948: The Dominican earthquake of August 1946. Bull. Seism.Soc.Amer., 38(1):1-32 (en Inglés).

MCNAMARA D.E., BENZ H., EARLE P., ODUM J., MCCARTHY J., PRESGRAVE B., WEAVER B., LEITH C., y Oppenheimer D. Capability analysis of a proposed nine station seismic network in the Caribbean. $17^{\text {th }}$ Caribbean Geological Conference, San Juan, Puerto Rico. July 19-21, 2005 (en Inglés).

MCNAMARA D.E., HILLEBRANDT C. VON Y CRUZ CALDERÓN G., 2005A: Nueva red sísmica en la región del Caribe, Golfo de México y la costa este del Atlántico para el sistema de alerta de tsunamis. Revista Ciencia y Tecnología, 16:18-22 (en Inglés).

MERCADO A. Y MCCANN W., 1998: Numerical simulation of the 1918 Puerto Rico tsunami. Natural Hazards, 18(1):57-76 (en Inglés).

MOLINA E. Tsunami catalogue for Central America 1539-1996. Technical Report No. II-104. Reduction of Natural Disasters in Central America. Institute of Solid Earth, University of Bergen, Norway, 1997 (en Inglés).

MONTERO W., 1990: Tsunamis en Costa Rica. Boletín del Colegio de Geólogos de Costa Rica.

MONTELIEU E., 1968: Notas y manuscritos (inéditos) del Ingeniero Don Eduardo Montelieu $(\dagger)$. Fondos del Departamento de Sismología, Instituto de Geofísica y Astronomía, Academia de Ciencias de Cuba.

MONTEULIEU E., 1933: Informe de la comisión nombrada para el estudio del terremoto de Santiago de Cuba de Febrero de 1932. Revista Sociedad Cubana de Ingenieros, 25(1):1-79.

MORALES Y PEDROSO L., 1931: Los terremotos en Cuba. Revista de la Sociedad Cubana de Ingenieros, XXIII(5):264-308.

NAGANO D., IMAMURA F. Y SHUTI N., 1991: A numerical model for field tsunamis and its implication to predict damages done to aquaculture. Natural Hazards, 4:235255 (en Inglés).

NEALON J.W. Y DILLON W.P. Earthquakes and tsunamis in Puerto Rico and the U.S. Virgin Islands. USGS Fact Sheet FS-141-00, April 2001 (en Inglés).

NG M.K.F., LE BLOND P.H. Y MURTY T.S., 1990: Simulation of tsunamis from great earthquakes on the Cascadia subduction zone. Science, 250:1,248-1,251 (en Inglés).

NOAA, 2004: National tsunami hazard mitigation program. [http://www.pmel.noaa.gov/tsunami/Dart] (en Inglés).

NUÑEZ-CORNÚ F.J., ORTIZ M. Y SÁNCHEZ J.J., 2008: The great 1787 Mexican tsunami. Nat. Hazards, 47:569-576 (en Inglés).

O'LOUGHLIN K.F. Y LANDER J.F. Caribbean tsunamis: A 500-year history from 14981998. Advances in Natural and Technological Hazards Research, vol. 20 Boston, MA: Kluwer Academic Publishers, 2003 (en Inglés).

POEY, A. Tableau chronologique des tremblements de terre resentís a l' ile de Cuba de 1551 à 1855. Annales des Voyages, $6^{\mathrm{a}}$ serie, 11, 301 p. Malte-Brun, Paris, 1855 (en Francés).

PROTTI M., GUENDEL F. Y MCNALLY K., 1994: The geometry of the Wadati-Benniof zone under southern Central America and its tectonic significance: Result from a high resolution local seismograph network. Phys. Earth Planet.Int., 84:271-287 (en Inglés).

REID H.F. Y TABER S., 1919: The Puerto Rico earthquakes of October - November 1918. Bull.Seism.Soc.Amer., 9(4):95-127 (en Inglés). 
ROBSON G.R., 1964: An earthquake catalogue for the eastern Caribbean. Bull.Seism.Soc.Amer., 54(2):785-832 (en Inglés).

RODRÍGUEZ M. Trayectoria de los huracanes de gran intensidad que han azotado a Cuba 1844-1985. En: Nuevo Atlas de Cuba, VI, 2.1., 1989.

RODRÍGUEZ J.E., 1983: Estudio del oleaje. Informe científico del Instituto de Meteorología, Academia de Ciencias de Cuba. 20 p.

RODRÍGUEZ FERRER M. Naturaleza y civilización de la grandiosa isla de Cuba. Imprenta J. Noguera, Madrid, 1876.

ROMERO G. Redes sismológicas y de monitoreo oceanográficos en el Caribe. Simposio regional sobre tsunami en el Mar Caribe. IOCARIBE, Subcomisión para el Caribe y regiones adyacentes, COI, UNESCO, 20-21 octubre, 2008.

RUBIO M., 1982: Ocurrencia de tsunamis en el Caribe. Investigaciones Sismológicas en Cuba, 2:170-180.

RUBIO M., COTILLA M. Y ÁLVAREZ L., 1994: Evidencias sobre la microplaca Gonave. Informe científico-técnico, p. 20. En: Archivo del Departamento de Sismología, Instituto de Geofísica y Astronomía, Academia de Ciencias de Cuba.

SAINTE-CLAIRE DEVILLE M.Ch., 1867: Sur le tremblement de terre du 18 november 1867 aux Antilles. Comptes Rendus, Acad.Sci., Paris, 65:1.110-1.114 (en Francés).

SATAKE K. (Editor). Tsunamis: Case studies and recent developments. Dodrecht, Springer, 2005 (en Inglés).

SCHWAB W.C., DANFORTH W.W., SCALON K.M. Y MASSON D.G., 1991: A giant submarine slope failure on the northern insular slope of Puerto Rico. Marine Geology, 96:237-246 (en Inglés).

SHUBERT C., 1994: Tsunamis in Venezuela: Some observations on their occurrence. Journal of Coastal Research. Special Issue, 13, Coastal Hazards, 189 (en Inglés).

SHUTO N., 1991: Numerical simulation of tsunamis. Its presents and near future. Natural Hazards, 4:171-191 (en Inglés).

SIGURDSSON H. Volcanic tsunami. Summary Report IOCARIBE Tsunami Warning Workshop, St. Johns, Virgin Islands. May, UNESCO IOC Workshop Report, Paris, 1996 (en Inglés).

TOMBLIN J.M. Y ROBSON G.R. A catalogue of felt earthquakes for Jamaica with references to other islands in the Greater Antilles: 1524-1971. Mines Geol.Div. Special Publ., 2, Jamaica, 1977 (en Inglés).

UNESCO-IOC. IUGG/IOC TIME Project: Numerical method of tsunami simulation with the leap-frog scheme. IOC Manuals and Guides No. 35. Paris, UNESCO, 1997 (en Inglés).

WEISSERT T.P., 1990: Tsunami travel time charts for the Caribbean. Science of Tsunami Hazard, 8(2):67-78 (en Inglés).

ZAHIBO N. Y PELINOVSKY E.N., 2001: Evaluation of tsunami risk in the Lesser Antilles. Natural Hazards and Earth System Sciences, 1:221-231 (en Inglés). 